\title{
Parents under-report children's suicide ideation and attempts
}

Breton JJ, Tousignant M, Bergeron L et al. Informant-specific correlates of suicidal behaviour in a community survey of 12 to 14 year olds. Journal of the American Academy of Child and Adolescent Psychiatry 2002 June; 41: 723-30.

\section{QUESTION: Are there differences in the correlates of suicidal ideation in early adolescence reported by children and their parents?}

Design

Cross sectional survey.

\section{Setting}

Quebec, Canada.

\section{Participants}

825 adolescents, aged $12-14$ years, and their parents. A weighted sample was used from the Quebec Child Mental Health Survey of 2400 children aged 6-14 years in 1992. Eligible children spoke French or English and had lived mainly with their parent(s) during the past 12 months. Children with sensory or mental handicaps and those living on reserves or in remote areas were excluded.

\section{Assessment of risk factors}

The adolescent and one parent (91\% mothers) were interviewed simultaneously in different rooms $(80 \%$ response rate). Adolescent, family and socioeconomic characteristics were assessed. Logistic regression was used to compare correlates of suicidality among youth and parents.

Sources of funding: Quebec Ministry of Health and Social Services.

For correspondence: JJ Breton,

Riviere-des-Prairies Hospital, Quebec,

Canada.

jj.breton.hrdp@

ssss.gouv.qc.ca

\section{Main outcome measures}

Suicide ideation within 6 months; suicide attempts within 6 months and during lifetime.

\section{Main results}

59 adolescents reported suicide ideation (7\%) and 36 reported suicide attempts (4\%). Parents identified 6 of these 59 suicide ideation reports $(10 \%)$ and 2 of the suicidality differed between parents and children (table). reported suicide attempts $(6 \%)$. Correlates of reported

Correlates of reported youth suicide ideation in past 6 months for adolescents and their parents

\begin{tabular}{lcc} 
Characteristics & $\begin{array}{c}\text { Adolescent report } \\
\text { odds ratio }\end{array}$ & $95 \%$ Cl \\
\hline Internalising disorders (1 disorder v none) & 3.1 & 1.1 to 8.3 \\
\hline Father's death & 4.4 & 1.3 to 14.5 \\
\hline Daily tobacco use & 5.4 & 2.2 to 13.3 \\
\hline Father's caring behaviours (high score v average) & 1.6 & 1.1 to 2.4 \\
\hline & $\begin{array}{c}\text { Parent report } \\
\text { odds ratio }\end{array}$ & $95 \%$ Cl \\
\hline Perceiving a need for help & 5.8 & 2.9 to 12.0 \\
\hline Parental depressive disorder & 3.0 & 1.5 to 6.2 \\
\hline Parental punitive behaviour (more v average score) & 1.5 & 1.1 to 2.0 \\
\hline 2 parent family v single parent & 1.3 & 0.5 to 3.0 \\
\hline 2 parent family v step parent family & 6.7 & 1.2 to 38.2 \\
\hline Adolescent admitted to institution & 6.6 & 1.5 to 28.7
\end{tabular}

Internalising mental disorders, stressful family events, regular tobacco use and parent-adolescent relationship difficulties predicted reported suicidality by adolescents. Correlates in the parental model included parental depressive disorders, parent-adolescent relationship difficulties, perceiving a need for help for the adolescent and institutionalising the child.

\section{Conclusions}

Correlates of youth suicidal behaviour differ between adolescents and parents. Informant-specific correlates of suicidality should be considered when developing research and interventions targeting youth suicide.

\section{COMMENTARY}

There are several reasons why assessing suicidality is more challenging than measuring most other indices of psychopathology. First, there is ambiguity in the definitions used to identify suicidality. Discrete behaviours that reflect unique suicidality constructs (for instance thoughts, gestures, self-mutilation, suicide attempts and so on) are often measured inconsistently. In addition, parents, youth and clinicians may have different ideas about whether self harmful thoughts and behaviours are 'suicidal'. Second, some forms of suicidality are difficult to detect by external reporters. Third, assessing suicidality involves unique ethical challenges. Investigators are limited to techniques that will maintain the anonymity of participants or allow easy access to the caretakers of at-risk youths.

Breton et al address some of these issues. Results suggest that parents may be poor informants of suicidality, underreporting their children's suicidal ideation and behaviour. Findings on the correlates of suicidality are more difficult to interpret, given the absence of theoreticallydriven risk models. Statistical procedures were not used to control for method variance and inflated experiment-wise error rates. Nevertheless, the findings could guide future research, including prospective studies of associations between family variables and suicidality that control for related factors and allow us to differentiate incidental predictors from etiologic predictors.

There is now a body of literature documenting informant-based differences in suicidality rates. Future research should advance our understanding of when and why reporting differences exist. We also need to explore how reporting discrepancies might be valuable in clinical evaluations and develop innovative assessment approaches to address the unique challenges of assessing suicidality.

Assistant Professor Mitchell J Prinstein and Matthew K Nock Department of Psychology Yale University, USA 